\title{
Effect of intrathecal glycine and related amino acids on the allodynia and hyperalgesic action of strychnine or bicuculline in mice
}

\author{
Eui Sung Lim, and II Ok Lee*
}

Department of Anesthesiology and Pain Medicine, Madi Hospital, *Department of Anesthesiology and Pain Medicine, Korea University College of Medicine, Seoul, Korea

Background: The intrathecal (IT) administration of glycine or GABA receptor antagonist result in a touch evoked allodynia through disinhibition in the spinal cord. Glycine is an inhibitory neurotransmitter that appears to be important in sensory processing in the spinal cord. This study was aimed to evaluate the effect of glycinerelated amino acids on antagonizing the effects of IT strychnine (STR) or bicuculline (BIC) when each amino acid was administered in combination with STR or BIC. Methods: A total of 174 male ICR mice were randomized to receive an IT injection of equimolar dose of glycine, betaine, $\beta$-alanine, or taurine in combination with STR or BIC. Agitation in response to innocuous stimulation with a von Frey filament after IT injection was assessed. The pain index in hot-plate test were observed after IT injection. The effect of IT muscimol in combination with STR or BIC were also observed. Results: The allodynia induced by STR was relieved by high dose of glycine or betaine. But, allodynia induced by BIC was not relieved by any amino acid. Whereas the STR-induced thermal hyperalgesia was only relieved by high dose of taurine at 120 min after IT injection, the BIC-induced one was relieved by not only high dose of taurine at $120 \mathrm{~min}$ but also low dose of glycine or betaine at $60 \mathrm{~min}$ after IT injection. The BICinduced allodynia and thermal hyperalgesia was relieved by IT muscimol. Conclusions: This study suggests that IT glycine and related amino acids can reduce the allodynic and hyperalgesic action of STR or BIC in mice. (Korean J Anesthesiol 2010; 58: 76 86)

\section{Key Words: Allodynia, Bicuculline, Glycine, Neuropathic pain model, Strychnine, Thermal hyperalgesia.}

\footnotetext{
Received: June 29, 2009. Revised: July 21, 2009. Accepted: July 21, 2009.

Corresponding author: II Ok Lee, M.D., Department of Anesthesiology and Pain Medicine, Korea University College of Medicine, 97, Gurodong-gil, Guro-gu, Seoul 152-703, Korea. Tel: 82-2-2626-1437, Fax: 82-2-851-9897, E-mail: iloklee@korea.ac.kr This paper is a thesis for a doctor's degree.

(c) This is an open-access article distributed under the terms of the Creative Commons Attribution Non-Commercial License (http:// creativecommons.org/licenses/by-nc/3.0/), which permits unrestricted non-commercial use, distribution, and reproduction in any medium, provided the original work is properly cited.
} 


\section{Introduction}

Allodynia is referred to as the state of discomfort and pain evoked by innocuous stimulations that normally cause no pain, and it is a typical symptom of neuropathic pain. One of the pathophysiologic mechanisms of allodynia is considered to be decreased inhibitory function of the posterior horn of spinal cord. Two representative inhibitory neurotransmitters that are associated with the posterior horn of spinal cord include glycine and $\gamma$-aminobutyric acid (GABA), both of which play a key role in processing low threshold stimuli within the spinal cord. These molecules increase the influx of $\mathrm{Cl}$ ion to the neurons by opening a ligand-gated ion channel and thereby induce the hyperpolarization of the cell membrane. Thus, the excitability of neurons is decreased $[1,2]$. Accordingly, an intra-thecal administration of strychnine (STR)sensitive glycine receptor antagonist (STR) or GABA $A_{A}$ receptor antagonist (bicuculline $[\mathrm{BIC}]$ ) provokes the disinhibition in the posterior horn of spinal cord. Owing to this, allodynia which sensitively responds to a mild stimulation occurs $[3,4]$.

Normally, a low-threshold stimulus $A_{-} \beta$ input is mainly controlled by glycine, one of the key inhibitory neurotransmitters of the posterior horn of spinal cord [5]. An intra-thecal administration of STR has an antagonistic effect on the STRsensitive glycine receptor on the post-synaptic membrane. This inhibitory control disappears. A synaptic connection between $A_{-}{ }_{\beta}$ fibers and the pain-signaling pathway is strengthened. Therefore, this stimulation is misrecognized as the pain [5]. Moreover, this increases the excitability of secondary neurons with the N-methyl-D-aspartate (NMDA) receptor on the post-synaptic membrane [4]. In contrast, an intra-thecal administration of $\mathrm{BIC}$ blocks $\mathrm{GABA}_{\mathrm{A}}$ receptor on the post-synaptic membrane as well as the pre-synaptic one of spinal dorsal horn. It is a matter of course that this phenomenon increases the reactivity transferring the responses to $\mathrm{A}_{\beta}$ input which is involved in innocuous stimulations. In other words, BIC inhibits the inhibitory activity of GABA on the pre-synaptic membrane. Thus, it increases the secretion of excitatory amino acid, glutamate, at the primary afferent nerve ending. On the post-synaptic membrane, it activates the pathway involved in NMDA receptor-nitric oxide synthase (NOS). It eventually strengthens the excitatory postsynaptic potentials (EPSPs) [6].

Such amino acids associated with glycine as betaine, $\beta$ alanine and taurine act on the STR-sensitive glycine receptor. Once these amino acids activate glycine receptor, this triggers the generation of inhibitory postsynaptic potentials (IPSPs). It has also been reported that an intra-thecal administration of these amino acids alleviated symptoms of allodynia due to STR to some extents [7]. At the present, however, as one of the analgesic agents activating glycine receptor, glycine is a key amino acid that plays a role in regulating the inhibitory neurotransmission. Nevertheless, its toxicity or side effects (respiratory distress, sedation or seizure) restrict the actual use of glycine in a clinical setting. According to an experiment which was performed by Ishikawa et al. [4], an intra-thecal administration of BIC increased the secretion of taurine as well as glutamate.

Given this background, we administered glycine-like amino acids and $G A B A_{A}$ receptor agonists (muscimol) concomitantly with STR or BIC to the intra-thecal space. Thus, we attempted to examine the effects of each receptor antagonist on allodynia occurring due to STR or BIC.

Besides, most of the previous experimental studies where the occurrence of allodynia was induced have been conducted to examine the adverse reactions of mechanical nociceptor due to the tactile sensation. In other words, these studies have been conducted to examine tactile allodynia which transmits a low-threshold stimulation mediated by $\mathrm{A}{ }_{\beta}$ fibers. In the current study, we examined whether thermal hyperalgesia occurred because of heat, detrimental stimulus mediated by unmyelinated C-fibers, as well as mechanical nociceptor. Furthermore, we also examined whether such amino acids as glycine and muscimol would have any effects on these phenomenon.

\section{Materials and Methods}

ICR male mice weighed approximately $22 \mathrm{~g}$ (MJ Ltd., Seoul, Korea) were used for the current study, 3-4 of which were bred in a single cage. Experimental animals were allowed to freely take foods and water. In these animals, the photoperiodism was maintained. Then, these animals were accomodated to the experimental environment. All the experimental procedures were conducted in compliance with the guidelines specified on The Ethics Board of The Animal Experiments.

In a transparent anesthetic induction box which was filled with the air (500 ml volume), experimental mice and a gauze immersed in a liquid sevoflurane (Abbott Lab., Kent, UK) were placed altogether. Using a volatile sevoflurane, the inhalation anesthesia was performed. When the mobility disappeared, mice were taken out and then asked to perform a voluntary respiration. Then, based on the methods of Hylden and Wilcox [8], the drugs were intrathecally administered. A $25 \mu$ l Hamilton syringe (Hamilton Co., NV, USA) in which a 30-guage, 1.5-inch needle (BD, Franklin Lakes, NJ, USA) was installed was inserted between $L 5$ and L6. Then, the tip of a needle was confirmed to be well placed in the spinal cavity using a tail flick of mice. Following this, experimental drugs were administered at a volume of $5 \mu \mathrm{l}$. After a syringe was pulled out, mice were transferred to a tube where they could freely move. Following this, the behavioral test and hot plate 
test were performed.

For a total of 20 minutes following the administration of drugs, two types of behaviors such as spontaneous agitation (SA) and touch evoked agitation (TEA) were monitored. The severity of SA was assessed based on such numeric grades 0,1 and 2 in cases in which mice voluntarily showed the abnormal behavior even in the absence of stimulation during a 20-minute period (Table 1). TEA was assessed at a 2-minute interval for the occurrence of allodynia after innocuous touches were given to the hind legs and tail using von Frey filament 4.17 (1.4 g force; Stoelting Co., Chicago, IL) during a 20-minute monitoring period. At this time, investigators who evaluate the occurrence of allodynia were blinded with the types of drugs which were administered. An assessment of TEA was also performed based on the numeric grades (Table 1). In each group, the results of behavioral test were expressed using SA scores, TEA scores and a sum of these two scores (total agitation, TA). SA scores were defined as a sum of scores which were recorded during a 20-minute monitoring period. TEA scores were defined as a sum of scores which were recorded at a 2-minute interval during a 20-minute monitoring period in the hind legs and tail. Following the administration of drugs, the overall process of behavioral test was recorded using a digital video camera recorder (Sony, Japan).

Subsequently to behavioral test, to monitor the changes of pain sensation to the heat, the test using a hot plate (Ugo Basile, Comerio, Italy) was performed. Experimental animals which completed behavioral test were placed in a plastic box whose floor was manufactured with a glass. Then, these animals were stabilized. Following this, the light emitted from a heat source on the floor of glass plate was irradiated to the sole of mice. After that, the time elapsed while mice perceived the thermal sensation due to this heat $\left(55^{\circ} \mathrm{C}\right)$ and then withdrew the legs (paw withdrawal latency [PWL]) automatically using an electronic timer. This avoidance response time was defined as pain threshold in hot-plate test (HPPT). In cases in which there was a lack of the responses,

Table 1. Assessment of Agitation Score

\begin{tabular}{ll}
\hline \multicolumn{2}{l}{ SA score } \\
\hline 0 & No considerable movement \\
1 & Mild squeaking \\
2 & Scratching and biting the leg or tail \\
\hline TEA score \\
\hline 0 & No response \\
1 & $\begin{array}{l}\text { Mild squeaking with attempts to move away from the } \\
\text { stroking filament }\end{array}$ \\
2 & $\begin{array}{c}\text { Vigorous squeaking evoked by the stroking filament, } \\
\text { biting at the filament and strong efforts to escape }\end{array}$ \\
\hline
\end{tabular}

SA: spontaneous agitation, TEA: touch evoked agitation, TEA was induced by von Frey filament. to prevent the occurrence of burn injury, a heat source was automatically turned off. To do this, a cut-off time was set at 30 seconds. Prior to the administration of drugs, HPPT was measured twice and the baseline mean value of HPPT (basal HPPT) was calculated accordingly. Following the administration of drugs, HPPT was measured at 30 minutes, 60 minutes, 90 minutes and 120 minutes. The results were expressed as the pain index in hot-plate test (HPPI) at 30 minutes, 60 minutes, 90 minutes and 120 minutes.

$$
\text { HPPI = HPPT / Basal HPPT }
$$

All the reagents were purchased from Sigma Chemical Co. (St. Louis, USA). Each reagent was dissolved using a saline and then diluted in an artificial cerebrospinal fluid (aCSF) to a desirable concentration at the time of experimental procedure. In all cases, the drugs were made to have a total volume of $5 \mu$ and then injected. To induce the occurrence of allodynia, the dose of STR and BIC which were administered was determined to be $0.3 \mu \mathrm{g}$ for STR (mw 334.4) and $0.01 \mu \mathrm{g}$ for BIC (mw 367.4) with reference to the previous reports [2,3]. To determine the alleviating effects of allodynia, four types of amino acids were used. The dose of these amino acids was determined through a preliminary experiment prior to the current experiment. According to a preliminary experiment, if the concentration of glycine exceeded $100 \mathrm{mM}$, such side effects as seizure and motor disturbance occurred in some cases. Accordingly in the current study, the administration dose of amino acids was not higher than $100 \mathrm{mM}$. In the current experiment, four types of amino acids were used as the same mole (equimolar dose; $33 \mathrm{mM}$ ). In more detail, the administration doses were $12.5 \mu \mathrm{g}$ for glycine (mw 75.1), 19.5 $\mu \mathrm{g}$ for betaine ( $\mathrm{mw}$ 117.1), $14.5 \mu \mathrm{g}$ for $\beta$-alanine (mw 89.1) and $20.5 \mu \mathrm{g}$ for taurine (mw 125.1). Besides, to compare the effects depending on the difference in the doses of amino acids, the experiment was repeatedly performed using doses (100 mM) which were three times higher than the initial dose. Also in cases of muscimol (mw 114.1), through a preliminary experiment, the concentration which does not cause a motor disturbance (paralysis) was determined to be $2.5 \mathrm{mM}$ and this corresponded to a dose of $1.5 \mu \mathrm{g}$. The concentrations of aCSF per $\mathrm{L}$ were determined to be $\mathrm{NaCl} 117 \mathrm{mmol}, \mathrm{KCl} 4.5 \mathrm{mmol}$, $\mathrm{CaCl}_{2} 2.5 \mathrm{mmol}, \mathrm{MgCl}_{2} 1.2 \mathrm{mmol}, \mathrm{NaHPO}_{4} 1.2 \mathrm{mmol}, \mathrm{NaHCO}_{3}$ $25 \mathrm{mmol}$ and dextrose $11.4 \mathrm{mmol}$.

In the current study, six mice were assigned to each group as shown below:

(1) The group where STR or BIC were solely administered

(2) The group where glycine, betaine, $\beta$-alanine or taurine were solely administered

(3) The group where STR or BIC were concomitantly administered with glycine, betaine, $\beta$-alanine or taurine

(4) The group where STR or BIC were concomitantly ad- 
ministered with muscimol

(5) The group where aCSF was administered

It was also examined whether there would be any side effects due to experimental drugs that might affect the results of behavioral test or hot plate test. Side effects include sedation, dyskinesia, motor nerve paralysis, seizure and death. To determine whether sedation occurred, mice were given the experimental drugs. Then, mice were placed in a rubber cylinder of $6 \mathrm{~cm}$ in height and $2.5 \mathrm{~cm}$ in width [9]. Following this, the time elapsed while mice descended to the inferior site of a cylinder. In the group where aCSF was administered, this time did not exceed four minutes. Accordingly in cases in which more than four minutes elapsed, the occurrence of sedation was reported. Dyskinesia was defined as cases in which a marked abdominal stretching appeared while the head and upper body trunk were shaken to the left and right directions [10].

Statistical analysis was performed using SigmaStat 3.0 (Systat Software Inc., Chicago, USA). An assessment of the results between the STR group, the BIC group and the aCSF group was made in comparison with muscimol using Student's t-test. A concomitant administration of STR or BIC with each amino acid was compared using one-way analysis of variance (ANOVA). In statistically significant cases, a multiple comparison was made using Holm-Sidak or Dunn methods. All the measurements were expressed as mean \pm SE (SE: standard errors). A value of $\mathrm{P}<0.05$ was considered statistically significant.

\section{Results}

\section{A single administration with STR or BIC}

Following an intra-thecal administration of STR or BIC in mice, on a behavioral test using von Frey filament (1.4 g), TEA scores were significantly increased in both groups as compared with the aCSF group (STR group: $\mathrm{P}<0.01$, BIC group: $P<0.01$ )(Fig. 1A). Besides, on a hot plate test, at 60 minutes following the administration of drugs, HPPI was significantly decreased as compared with the aCSF group (STR group: $P=0.02, \mathrm{BIC}$ group: $P=0.03$ ) (Fig. $1 \mathrm{~B}$ )

\section{A single administration of glycine-like amino acids}

Following an intra-thecal administration of each amino acid including glycine, at both doses of $33 \mathrm{mM}$ and $100 \mathrm{mM}$, as compared with the aCSF group, there were no significant differences on a behavioral test and a hot plate test (Fig. 2). In the group where betaine and ${ }_{\beta}$-alanine were administered at a concentration of $100 \mathrm{mM}$, the sedation was observed until four minutes following the administration of drugs in each mice.

\section{A concomitant administration of STR or BIC with amino acids}

A behavioral test and a hot plate test following a concomitant administration of STR with amino acids: In cases in which amino acids including glycine were concomitantly administered with STR, at a dose of $33 \mathrm{mM}$, there were no significant differences on a behavioral test and a hot plate

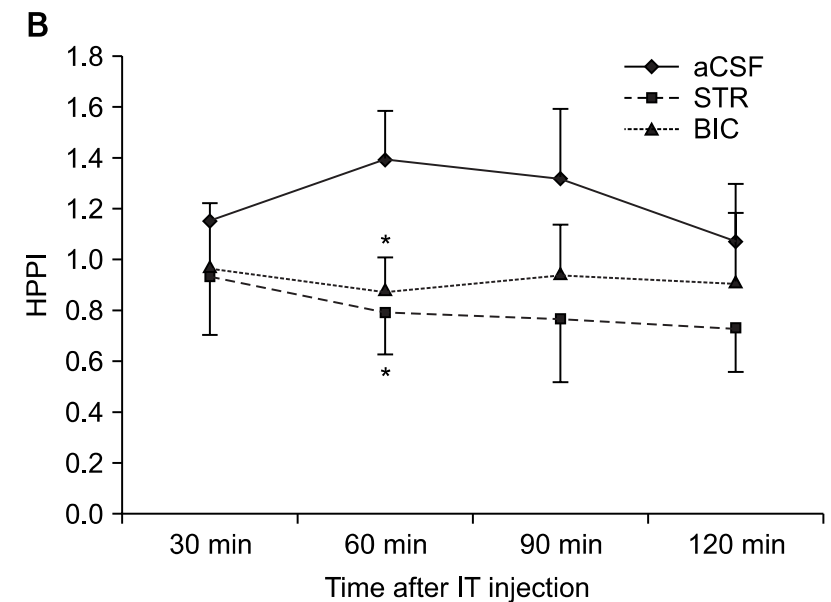

Fig. 1. Effect of the intrathecal (IT) injection of strychnine (STR) or bicuculline (BIC) on the agitation score induced by von Frey filament stimulation (A) and on the pain index in hot-plate test (HPPI) (B). Assessment of agitation score and HPPI were made as described in materials and methods. IT administration of STR or BIC in mice increased a sum of agitation score (A) and lowered on the HPPI at 60 min after IT injection (B) compared with artificial cerebrospinal fluid (aCSF) group. Data are expressed as the mean \pm SEM. *P $<0.05$ compared with aCSF group. TEA: touch evoked agitation, SA: spontaneous agitation, TA: total agitation. 
A

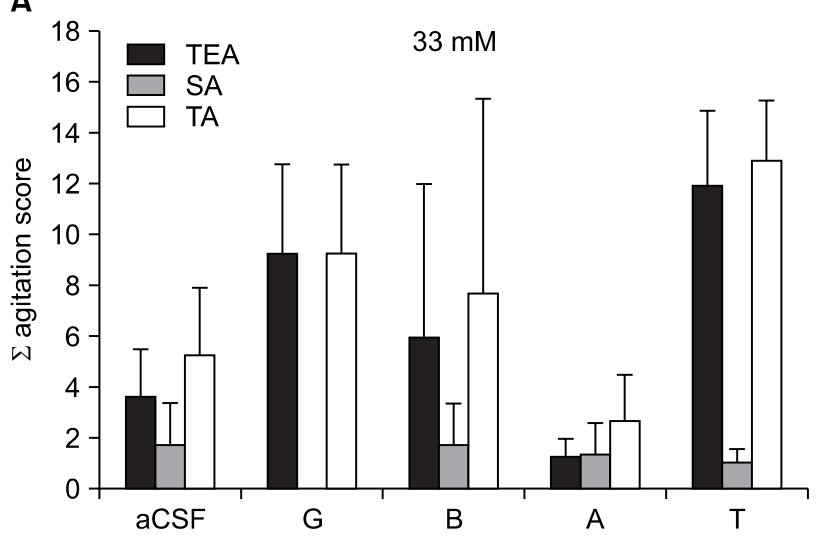

C

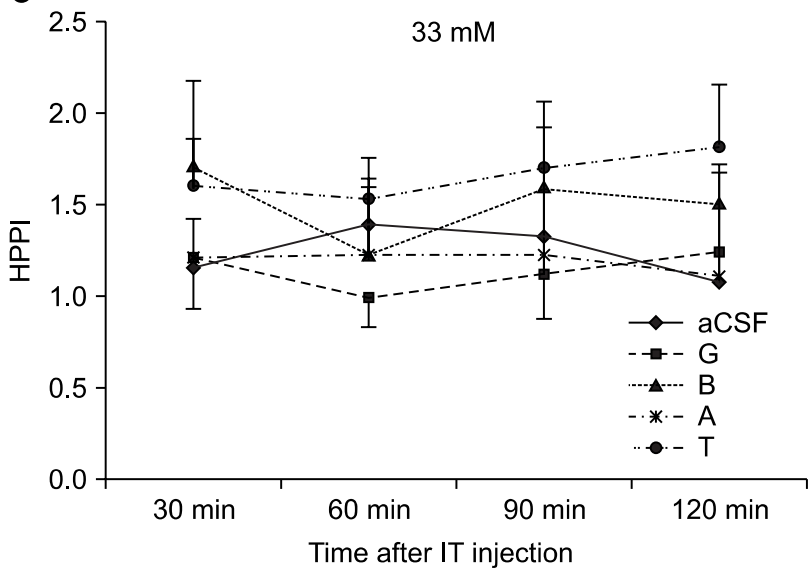

B

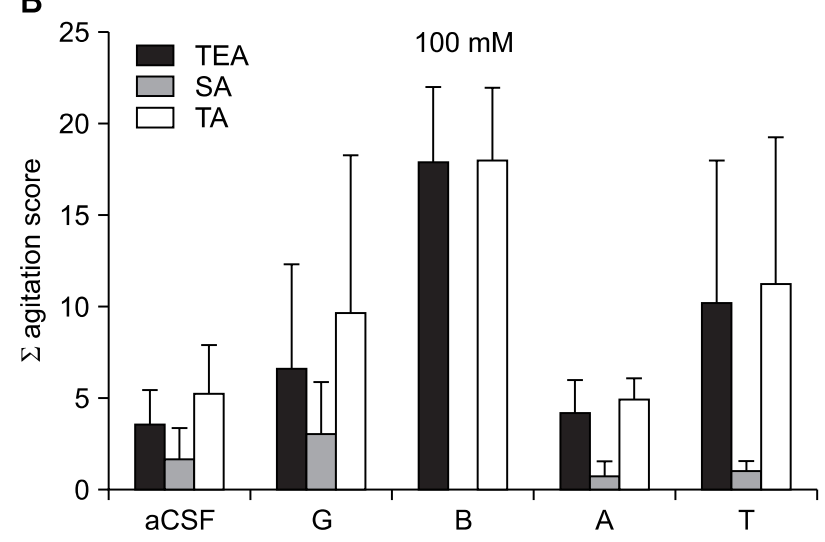

D

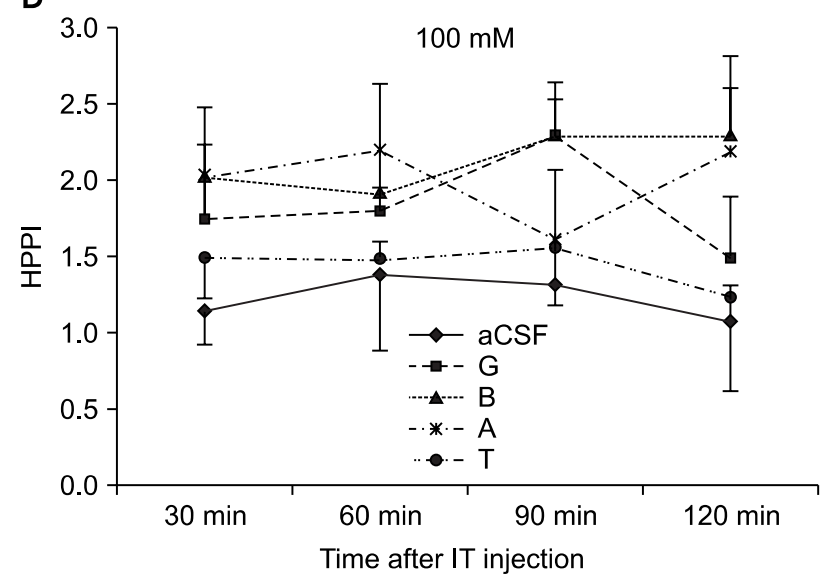

Fig. 2. Effect of the intrathecal (IT) injection of equimolar doses ( $33 \mathrm{mM}$ or $100 \mathrm{mM}$ ) of glycine $G$, betaine $B, \beta$-alanine $A$ or taurine $T$ on the agitation score induced by von Frey filament stimulation (A, B) and on the pain index in hot-plate test (HPPI) (C, D). Assessment of agitation score and HPPI were made as described in materials and methods. A sum of agitation score and the HPPI were not different by IT administration of $G, B$, A or T in mice compared with artificial cerebrospinal fluid (aCSF) group. Data are expressed as the mean \pm SEM. TEA: touch evoked agitation, SA: spontaneous agitation, TA: total agitation.

test (Fig. 3A and 3C). In cases in which amino acids $100 \mathrm{mM}$ were concomitantly administered with STR, however, TEA scores were significantly decreased in the group where glycine $(P<0.01)$ and betaine $(P<0.01)$ were concomitantly administered as compared with the group where STR was solely administered (Fig. 3B). On a hot plate test, however, HPPI was significantly increased in the group where taurine $33 \mathrm{mM}$ was concomitantly administered with STR as compared with STR was solely administered at 120 minutes following the administration of drugs ( $P<0.01$ ) (Fig. 3D).

A behavioral test and a hot plate test following a concomitant administration of BIC with amino acids: In cases in which amino acids including glycine were concomitantly administered with $\mathrm{GABA}_{\mathrm{A}}$ receptor antagonists $(\mathrm{BIC})$, at both doses of $33 \mathrm{mM}$ and $100 \mathrm{mM}$, there were no significant differences on a behavioral test (Fig. 4A and 4B). By contrast, on a hot plate test, following an experiment where amino acids $33 \mathrm{mM}$ were concomitantly administered with BIC, in the group where glycine $(P=0.01)$ and betaine $(P=0.03)$ were concomitantly administered, as compared with the group where BIC was solely administered at 60 minutes following the administration of drugs, HPPI was significantly increased (Fig. 4C). Besides, following an experiment using a dose of $100 \mathrm{mM}$, in the group where taurine and BIC were concomitantly administered, as compared with the group where BIC was solely administered at 120 minutes following the administration of drugs, HPPI was significantly increased ( $P$ $=0.02$ ) (Fig. 4D).

\section{A concomitant administration of STR or BIC with muscimol}

Following a concomitant administration of $\mathrm{GABA}_{\mathrm{A}}$ receptor agonist (muscimol) with STR, there were no significant differences on both a behavioral test and a hot plate test as compared with the group where STR was solely administered (Fig. 5A and 5B). In cases in which BIC was concomitantly 
A
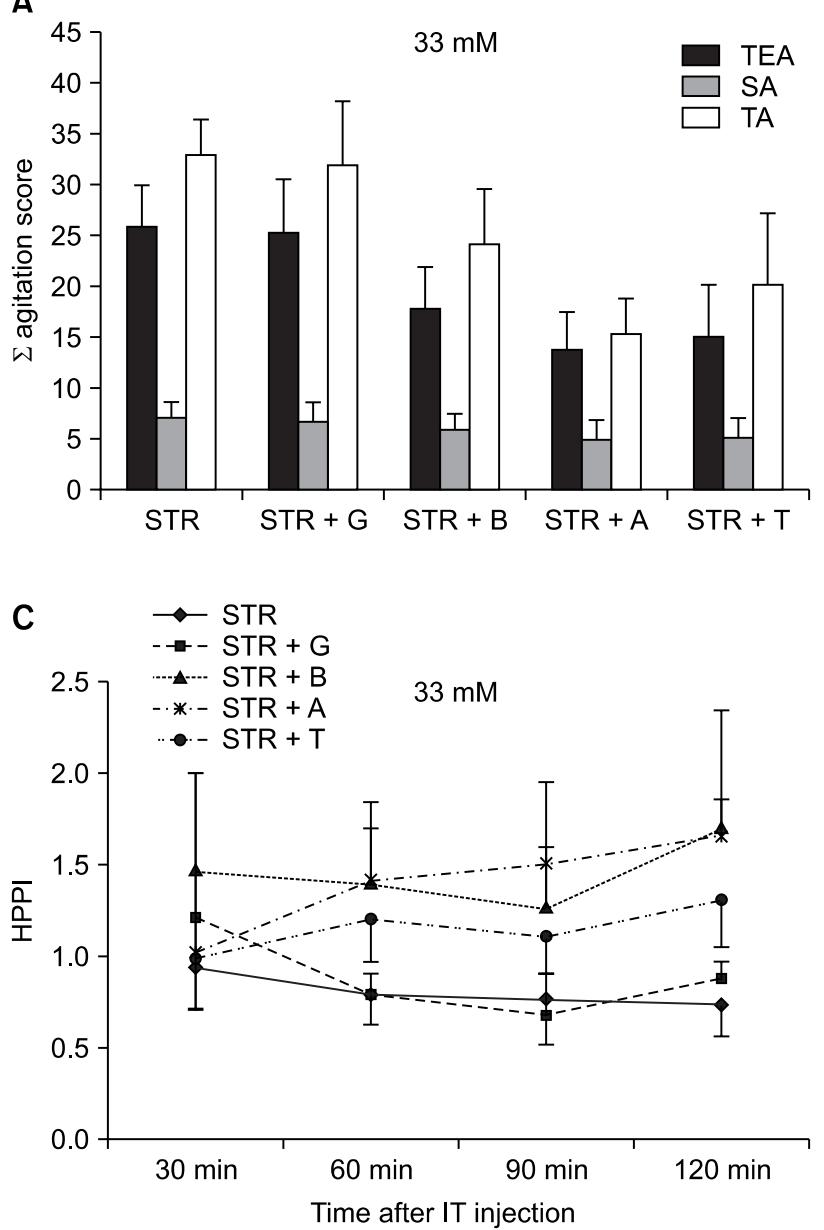

B
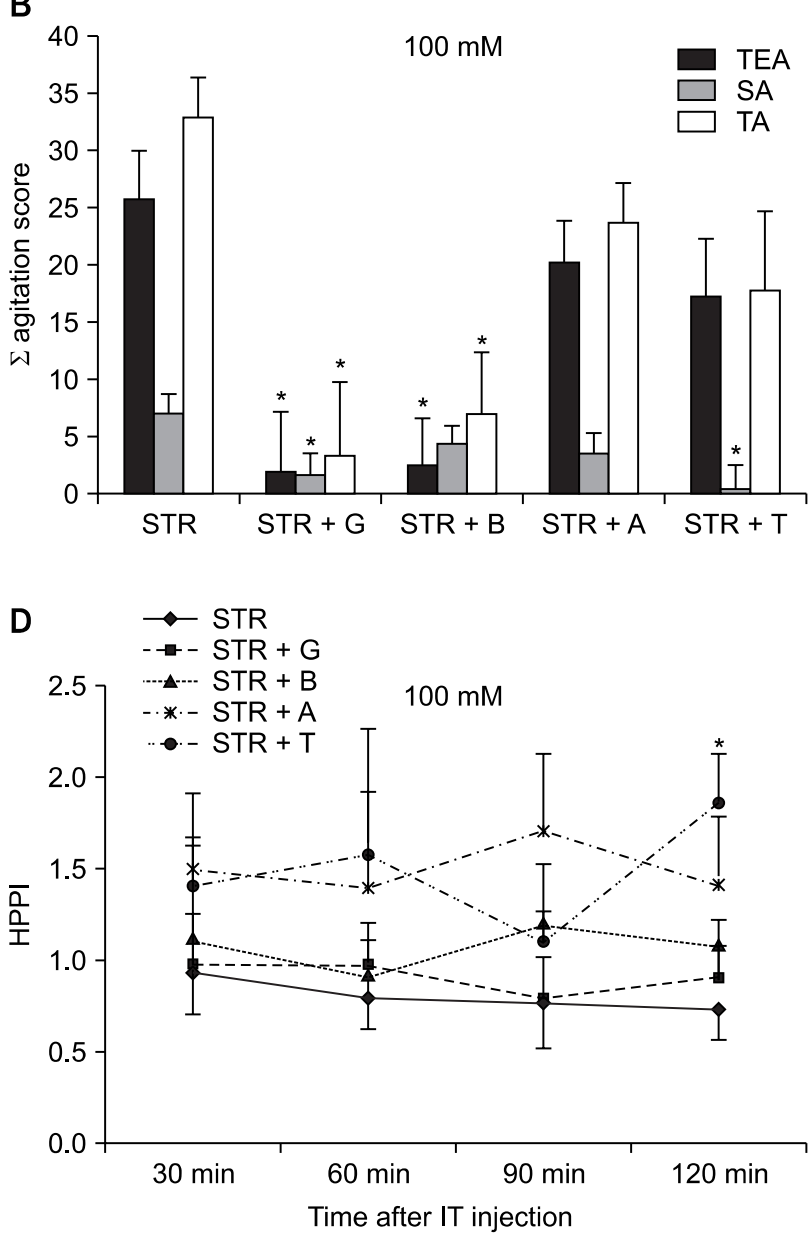

Fig. 3. Effect of the combined intrathecal (IT) injection of strychnine (STR) with equimolar doses ( $33 \mathrm{mM}$ or $100 \mathrm{mM}$ ) of glycine $\mathrm{G}$, betaine $B$, $\beta$-alanine A or taurine T on the agitation score induced by von Frey filament stimulation (A, B) and on the pain index in hot-plate test (HPPI) (C, D). Assessment of agitation score and HPPI were made as described in materials and methods. Combined IT STR with high dose of G or B in mice decreased a sum of agitation score compared with STR group (B). Combined IT STR with high dose of T increased on the HPPI at 120 min after IT injection compared with STR group (D). Data are expressed as the mean \pm SEM. ${ }^{*} \mathrm{P}<0.05$ compared with STR group. TEA: touch evoked agitation, SA: spontaneous agitation, TA: total agitation.

administered, as compared with the group where BIC was solely administered, TEA scores were significantly decreased on a behavioral test $(P=0.03)$ (Fig. 5A). On a hot plate test, at 60 minutes following the administration of drugs, HPPI was significantly increased ( $P=0.02$ ) (Fig. 5C).

\section{Discussion}

In the current study, STR (the antagonist for the representative inhibitory neurotransmitter in the posterior horn of spinal cord, glycine) and BIC (GABA antagonist) were intrathecally administered in mice. Thus, it showed the occurrence of allodynia and hyperalgesia. Through a intra-thecal concomitant administration of glycine-related amino acids (the agonists for glycine receptors) and muscimol (GABA receptor agonist) with STR or BIC, a partial alleviation of al- lodynia and hyperalgesia was confirmed to occur.

Allodynia that recognizes the innocuous stimulations as the pain and hyperalgesia that further perceives the pain sensation are major symptoms of neuropathic pain along with the spontaneous pain. This neuropathic pain shows a treatment challenge to clinicians due to an insufficient extent of the understanding of pathophysiology. Accordingly, to make a clear understanding of the pathophysiology of neuropathic pain, various types of animal experimental models have been developed. These include the spinal cord injury due to spinal hemisection [11], spinal contusion injury [12], chronic constriction injury [13], excitotoxic injury [14] and ischemic injury [15]. Most of these models have shown a behavioral response to the mechanical allodynia and the hyperexcitability of neurons existing in the posterior horn of spinal cord. Due to a loss of the glycine or the decreased 
A
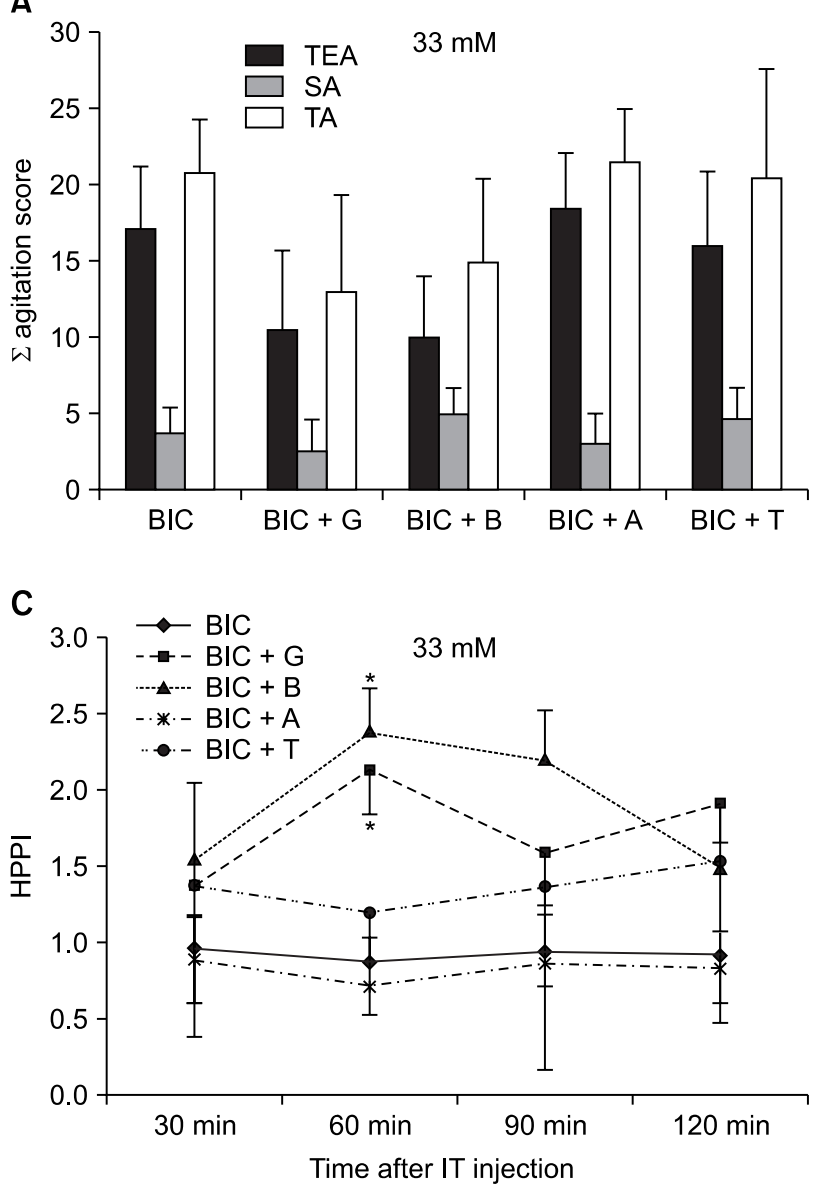

B

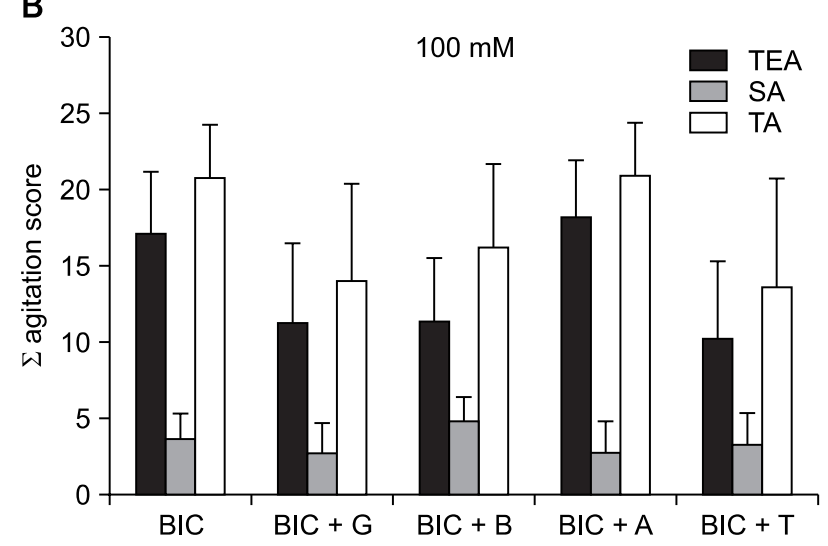

D

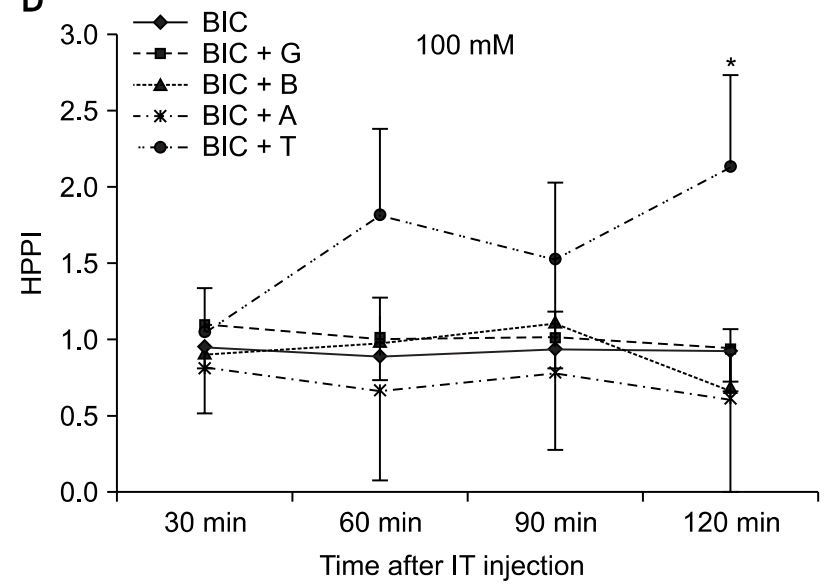

Fig. 4. Effect of the combined intrathecal (IT) injection of bicuculline (BIC) with equimolar doses (33 mM or $100 \mathrm{mM}$ ) of glycine $\mathrm{G}$, betaine $B, \beta$-alanine $A$ or taurine $T$ on the agitation score induced by von Frey filament stimulation $(A, B)$ and on the pain index in hot-plate test (HPPI) (C, D). Assessment of agitation score and HPPI were made as described in materials and methods. A sum of agitation score were not different by combined IT BIC with G, B, A or T compared with BIC group (A, B). Combined IT BIC with low dose of G or B in mice increased on the HPPI at 60 min after IT injection compared with BIC group (C). Combined IT BIC with high dose of T also increased on the HPPI at 120 min after IT injection (D). Data are expressed as the mean \pm SEM. *P $<0.05$ compared with BIC group. TEA: touch evoked agitation, SA: spontaneous agitation, TA: total agitation.

synthesis or secretion of GABA, the blockage of receptors and a loss of inhibitory intermediate neurons, if a loss of the inhibitory function should occur in the posterior horn of spinal cord, i.e., disinhibition should occur, allodynia would occur. This condition is characterized by the recognition of $\mathrm{A}_{\beta} \beta$ input of a low-threshold stimulus as the pain $[3,4]$. Therefore, a pharmacological experimental model where STR or BIC was intrathecally administered and thereby disinhibition was induced accordingly has been proposed.

Glycine and GABA are inhibitory neurotransmitters, which mediate the fast synaptic inhibition in the nervous system [1]. If these molecules should bind to their respective receptors, the influx of $\mathrm{Cl}$ ions would occur in neurons. Following the synapse formation, the hyperpolarization occurs and this leads to the decreased excitability of neurons [2]. In normal rats which received a light touch like a hair, the stimulus is transferred via $\mathrm{A}_{-}{ }_{\beta}$ fibers to the posterior horn of spinal cord. Due to the inhibitory functions of the posterior horn of spinal cord, there can be a response that the stimulated areas are simply gazed. In cases in which STR or BIC were administered intrathecally, however, this inhibitory function disappeared. This led to a hypersensitive behavior such as scratching, biting and crying in response to a light touch $[16,17]$. This is because at the terminal part of primary afferent neurons, the functions of GABA inhibiting the secretion of glutamate, one of the excitatory amino acids, are restricted. On the post-synaptic membrane, the pathway for NMDA receptor-NOS is activated [6]. According to Sherman and Loomis [18], an intra-thecal administration of STR triggered the occurrence of allodynia in a reversible, dose-dependent manner in rats. Also in the current study, following an intrathecal administration of STR or BIC, the occurrence of al- 
A

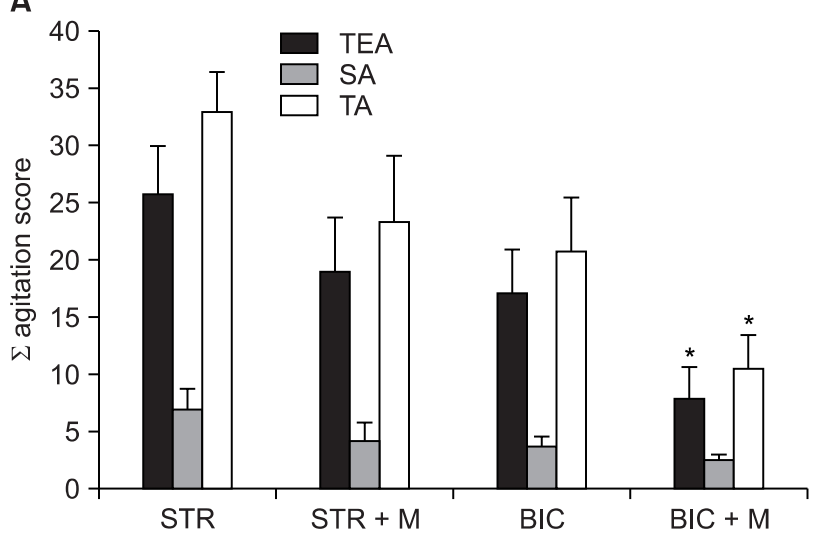

C

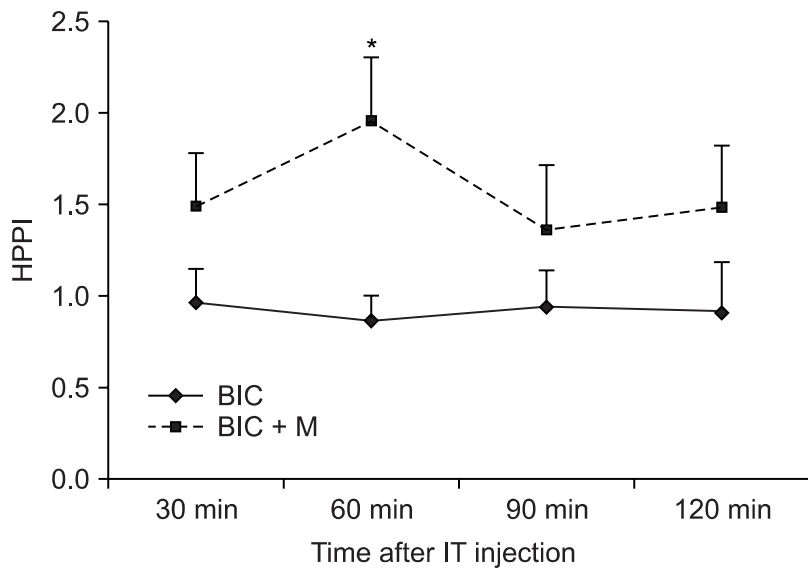

Iodynia response was found to be present in response to a light touch using a von Frey filament $(1.4 \mathrm{~g})$. Most of the previous studies using a pharmacological neuropathic pain model have mainly focused on the occurrence of allodynia via $A_{-} \beta$ fibers which are involved in transmitting a low-threshold mechanical stimulus. It therefore remains unclear whether hyperalgesia occurring via unmyelinated C-fibers is also associated with these models. Sherman and Loomis [19] induced the occurrence of allodynia following an intrathecal administration of STR. According to these authors, however, using a tail immersion in a $55^{\circ} \mathrm{C}$ water, there was a lack of the thermal hyperalgesia. According to Beyer et al. [20], following an intra-thecal administration of STR in rats, on a tail shock vocalization test, the threshold was significantly decreased and this led to the occurrence of hyperalgesia. In the current study, following an intra-thecal administration of STR in mice, PWL was diminished in response to a $55^{\circ} \mathrm{C}$ heat and this led to the occurrence of thermal hyperalegsia. As described here, the reasons that the results were different are that there was a discrepancy between the assessment methods in each experiment. Besides, the above differences might also be associated with the different doses per weight
B

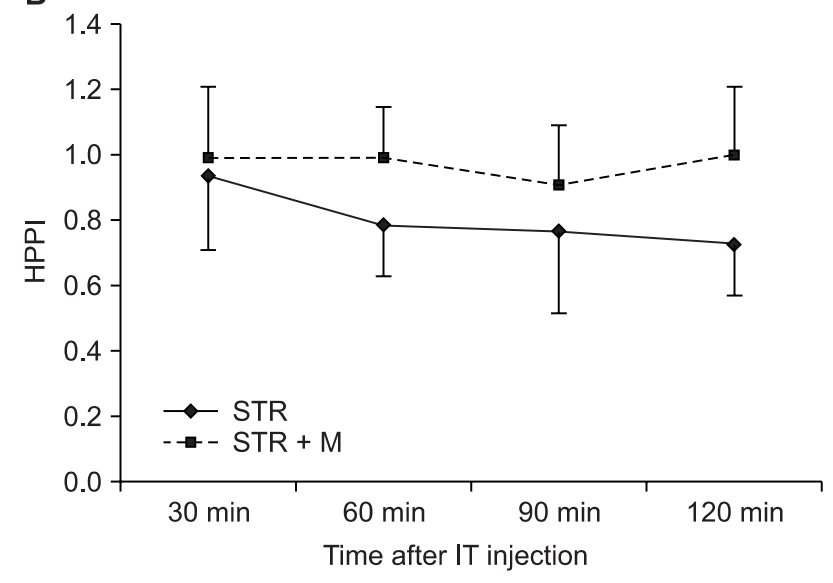

Fig. 5. Effect of the combined intrathecal (IT) injection of muscimol (M) with either strychnine (STR) or bicuculline (BIC) on the agitation score induced by von Frey filament stimulation (A) and on the pain index in hot-plate test (HPPI) $(\mathrm{B}, \mathrm{C})$. Assessment of agitation score and HPPI were made as described in materials and methods. A sum of agitation score and the HPPI were not different by combined IT $M$ with STR in mice compared with STR group(A, B). Combined IT $M$ with BIC decreased a sum of agitation score $(A)$ and increased on the HPPI at 60 min after IT injection compared with BIC group (C). Data are expressed as the mean \pm SEM. ${ }^{*} P<0.05$ compared with BIC group. TEA: touch evoked agitation, SA: spontaneous agitation, TA: total agitation.

which were used in the experiments. According to previous studies using Sprague-Dawley (SD) mice, the doses of 0.08 $-0.13 \mu \mathrm{g} / \mathrm{g}$ (body weight) [19] and $0.07-0.1 \mu \mathrm{g} / \mathrm{g}$ (body weight) [20] were used. In the current study where mice were used, however, the dose of approximately $0.015 \mu \mathrm{g} / \mathrm{g}$ (body weight) was used.

Such amino acids associated with glycine as betaine, $\beta$ -alanine and taurine can replace STR which bound to STRsensitive glycine receptor. It is therefore expected that this would compensate the effects due to STR [21]. Accordingly in the current study, following an intra-thecal administration of STR concomitantly with glycine, betaine, $\beta$-alanine or taurine at doses of $33 \mathrm{mM}$ and $100 \mathrm{mM}$, it was examined whether this concomitant administration would have an alleviating effect on allodynia and hyperalgesia. On the other hand, GABA has a similar anatomical location to glycine within the spinal cord. Besides, glycine and $\mathrm{GABA}_{\mathrm{A}}$ receptor exist on the same post-synaptic membrane. Therefore, these two molecules are responsible for the inhibitory functions in the posterior horn of spinal cord in a reciprocal manner [3]. In the pathophysiology by which allodynia occurs due to STR or BIC, glutamate receptor and NOS pathway are closely related [1]. 
It was therefore examined whether these amino acids have an effect on allodynia and allodynia due to BIC as well as STR. As a result, glycine and betaine significantly alleviated the symptoms of allodynia due to STR at a dose of $100 \mathrm{mM}$. On a hot plate test which was performed to assess the occurrence of hyperalgesia, however, no statistically significant effects were found. These results were in agreement with the previous reports using SD rats that there were no significant effects on a tail shock test which was performed to assess thermal hyperalgesia although an intra-thecal administration of glycine alleviated the occurrence of allodynia due to STR [20]. Besides, Sherman and Loomis [7] also reported that an intra-thecal administration of glycine and betaine suppressed the occurrence of allodynia due to STR in a dose-dependent manner. Following an administration of glycine and betaine, however, no allodynia due to BIC was alleviated. This implies that IPSPs induced due to GABA as compared with glycine was maintained during a long-term period [3]. Because glycine was very promptly cleared by a high-affinity uptake system whose Michaelis constant (Km) was 26.5-121 $\mu \mathrm{M}$ in the spinal cord [22], a concomitant administration with BIC would not produce a sufficient extent of the effects.

$\beta$-alanine has a lower degree of the binding activity to STRsensitive glycine receptor as compared with glycine and a higher degree of the binding activity to STR-sensitive glycine receptor as compared with taurine [23]. Besides, as shown in glycine, $\beta$-alanine also has a low-affinity uptake system [7]. Accordingly in the current experiment, there were no significant alleviating effects in both allodynia and thermal hyperalgesia due to STR or BIC. Besides, according to Beyer et al. [20], a concomitant administration of $\beta$-alanine with STR further increased such behaviors as scratching or biting of rats in a different manner from other amino acids. This phenomenon was explained by the fact that $\beta$-alanine also acted on STR-binding sites which were very closely located to glycine-binding sites of glycine receptor.

Taurine did not show a significant effect in alleviating allodynia due to STR. Taurine has a very low degree of the affinity to STR-sensitive glycine receptor as compared with other amino acids, and it has approximately 1/3 of effectiveness as compared with glycine [23]. Owing to this, as compared with glycine, taurine has a lower degree of the efficacy in alleviating allodynia due to STR. At 120 minutes following the administration of drugs, however, taurine suppressed the occurrence of thermal hyperalgesia due to STR or BIC at a concentration of $100 \mathrm{mM}$. Considering that the action time of STR or BIC which were intrathecally administered did not exceed one hour $[4,7]$, this can be considered an effectiveness of taurine itself. This is because taurine can control the activity of GABA, suppresses an influx of Ca ions, lowers the excitability of neurons, has a very low intake system as compared with glycine or $\beta$-alanine and acts on glycine receptor during a long-term period [20]. Besides, it should also be referenced that an intra-thecal release of taurine was significantly increased following an intra-thecal administration of BIC [4].

In normal rats, the blockage of GABA receptor of spinal cord promoted C-fiber inducing reactions in the posterior horn of spinal cord and it also induces the occurrence of mechanical allodynia [22,23]. In experimental rats whose peripheral nerve was damaged, the apoptosis of neurons occurs. This causes a functional loss of GABA-related neuronal transmission in the posterior horn of spinal cord [26]. Hwang and Yaksh [27] administered muscimol, one of the $\mathrm{GABA}_{\mathrm{A}}$ receptor agonists, via an intra-thecal route and thereby alleviated symptoms of mechanical allodynia which was induced by the damage of peripheral nerve. This is because muscimol increases the permeability to $\mathrm{Cl}$ ions and thereby causes a hyperpolarity of nerves following the synapse [28]. In the current study, following an intra-thecal administration of muscimol 1.5 $\mu \mathrm{g}$, as expected, allodynia and thermal hyperalgesia due to BIC were alleviated. But this showed no significant effects in alleviating allodynia and thermal hyperalgesia due to STR. By contrast, Loomis et al. [3] performed an experiment using SD rats. According to these authors, following an intrathecal administration of muscimol, allodynia due to STR was alleviated in a dose-dependent manner. But there was a slight discrepancy in the experimental methods from the current study. In the current study, following a one-time concomitant administration of STR and muscimol, allodynia was assessed based on the abnormal behavior in mice which were mentally alerted. According to Loomis et al. [3], however, while the changes in heart rate and blood pressure were monitored, an intravenous anesthesia was performed urethane using STR was administered at 15 minutes after a catheter was placed in the subarachnoid space and muscimol was administered. As described herein, there were changes in the methods for assessing allodynia.

As a result of the current study, a pharmacological model of neuropathic pain model was established using STR or BIC. This model triggered the occurrence of drug-induced disinhibition in the posterior horn of spinal cord and thereby induced the occurrence of allodynia and hyperalgesia, both of which were major symptoms of the neuropathic pain. According to the previous mechanisms by which the nerve of experimental rats was damaged [11-15], several days to several weeks elapsed until allodynia and hyperalgesia occurred following the onset of nerve damage. Besides, as a result of nerve damage, the somatosensory dysfunction occurred. This leads to various changes in the plasticity and functions of neurons and these include injury discharge, neuronal sprouting, the synthesis and degradation of neurotransmitters and apoptosis [18]. By contrast, because a pharmacological pain model directly acted on neurotransmitters, it can 
be established promptly. Moreover, because it causes no irreversible functional changes or morphology of neurons, its use impinges no ethical guidelines. Furthermore, it can be repeatedly reconstructed. By contrast, most cases of the neuropathic pain are persistently present during a chronic period. In comparison with this, the action time of this model was extremely short. It therefore remains problematic that studies cannot be conducted during a sufficient length of the period. Besides, because STR or BIC were concomitantly administered with other drugs (glycine etc.), the occurrence of resulting problems could not be completely ruled out. Furthermore, limitations of the current study are as follows:

(1) Allodynia was measured for 20 minutes following the administration of drugs.

(2) A hot plate test was performed during a period ranging from 30 to 120 minutes.

Owing to these limitations, there are problems that the timedependent differences are present in the assessment of two symptoms of neuropathic pain. Moreover, the action time of STR or BIC which were intrathecally administered was shorter than that of a hot plate test. Accordingly, the possibility that the effects of several amino acids in alleviating hyperalgesia were not accurately evaluated could not also be completely ruled out.

In cases of neuropathic pain, there is a lack of the sufficient understanding of its pathophysiology. It cannot be well treated with general types of analgesia such as non-steroidal anti-inflammatory drugs or opioids. It is therefore imperative that novel types of analgesics be developed promptly. According to a pain model using STR as shown in the current study, glycine and betaine reduced the occurrence of allodynia in a dose-dependent manner. Based on the alleviating effects of inhibitory amino acids as described herein, the development of treatment agents would be possible. Because glycine has a strong polarity at a physiological $\mathrm{pH}$ as well as toxicity or side effects, however, it discloses disadvantages that it has a poor degree of the permeability to the blood-brain barrier. Further studies are therefore warranted to overcome this. On the other hand, various amino acids including glycine showed no effects in alleviating allodynia due to BIC. In addition, muscimol also showed no effects in alleviating allodynia and hyperalgesia due to STR. These results indicate that the administration of antagonists for different types of receptors was of insignificance. Furthermore, further detailed studies are warranted to examine the effects of taurine in alleviating hyperalgesia due to STR and BIC.

In conclusion, following an intra-thecal administration of STR or BIC in mice, a neuropathic pain model could be established promptly. In association with this, further studies are warranted to examine the dose, action time, the pattern of administration and its methods. This would be helpful for developing novel types of analgesics based on the alleviation of disinhibition.

\section{Acknowledgements}

We would like to thank Jae-Woong Han for helpful advice and technical supports.

\section{References}

1. Onaka M, Minami T, Nishihara I, Ito S. Involvement of glutamate receptors in strychnine- and bicuculline-induced allodynia in conscious mice. Anesthesiology 1996; 84: 1215-22.

2. Chen Y, Dai TJ, Zeng YM. Strychnine-sensitive glycine receptors mediate analgesia induced by emulsified inhalation anaethetics in thermal nociception but not in chemical nociception. Basic Clin Pharmacol Toxicol 2007; 100: $165-9$

3. Loomis CW, Khandwala H, Osmond G, Hefferan MP. Coadministration of intrathecal strychnine and bicuculline effects synergistic allodynia in the rat: an isobolographic analysis. J Pharmacol Exp Ther 2001; 296: 756-61.

4. Ishikawa T, Marsala M, Sakabe T, Yaksh TL. Characterization of spinal amino acid release and touch-evoked allodynia produced by spinal glycine or $\mathrm{GABA}_{\mathrm{A}}$ receptor antagonist. Neuroscience 2000; 95: 781-6.

5. Khandwala H, Loomis CW. Milacemide, a glycine pro-drug, inhibits strychnine-allodynia without affecting normal nociception in the rat. Pain 1998; 77: 87-95.

6. Lee YW, Ishikawa T. Effects of agmatine on $\mathrm{GABA}_{\mathrm{A}}$ receptor antagonist-induced tactile allodynia. Korean J Pain 2008; 21: 173-8.

7. Sherman SE, Loomis CW. Strychnine-dependent allodynia in the urethane-anesthetized rat is segmentally distributed and prevented by intrathecal glycine and betaine. Can J Physiol Pharmacol 1995; 73: 1698-705.

8. Hylden JL, Wilcox GL. Intrathecal morphine in mice: a new technique. Eur J Pharmacol 1980; 67: 313-6.

9. Porreca F, Mosberg HI, Omnaas JR, Burks TF, Cowan A. Supraspinal and spinal potency of selective opioid agonists in the mouse writhing test. J Pharmacol Exp Ther 1987; 240: 890-4.

10. Larson AA. Intrathecal GABA, glycine, taurine or betaalanine elicits dyskinetic movements in mice. Pharmacol Biochem Behav 1989; 32: 505-9.

11. Gwak YS, Nam TS, Paik KS, Hulsebosch CE, Leem JW. Attenuation of mechanical hyperalgesia following spinal cord injury by administration of antibodies to nerve growth factor in the rat. Neurosci Lett 2003; 336: 117-20.

12. Siddall $P, X u C L$, Cousins M. Allodynia following traumatic spinal cord injury in the rat. Neuroreport 1995; 6: 1241-4. 
13. Vissers K, Adriaensen H, De Coster R, De Deyne C, Meert TF. A chronic-constriction injury of the sciatic nerve reduces bilaterally the responsiveness to formalin in rats: a behavioral and hormonal evaluation. Anesth Analg 2003; 97: 520-5.

14. Yezierski RP, Liu S, Ruenes GL, Kajander KJ, Brewer KL. Excitotoxic spinal cord injury: behavioral and morphological characteristics of a central pain model. Pain 1998; 75: 141 55.

15. Xu XJ, Hao JX, Aldskogius H, Sieger A, Wiesenfeld-Hallin Z. Chronic pain-related syndrome in rats after ischemic spinal cord lesion: a possible animal model for pain in patients with spinal cord injury. Pain 1992; 48: 279-90.

16. Yaksh TL. Behavioral and autonomic correlates of the tactile evoked allodynia produced by spinal glycine inhibition: effects of modulatory receptor systems and excitatory amino acid antagonists. Pain 1989; 37: 111-23.

17. Sivilotti $L$, Woolf $C J$. The contribution of $G A B A_{A}$ and glycine receptors to central sensitization: disinhibition and touch-evoked allodynia in the spinal cord. J Neurophysiol 1994; 72: 169-79.

18. Sherman SE, Loomis CW. Morphine insensitive allodynia is produced by intrathecal strychnine in the lightly anesthetized rat. Pain 1994; 56: 17-29.

19. Sherman SE, Loomis CW. Strychnine-sensitive modulation in selective for non-noxious somatosensory input in the spinal cord of the rat. Pain 1996; 66: 321-30.

20. Beyer C, Banas C, Gomora P, Komisaruk BR. Prevention of the convulsant and hyperalgesic action of strychnine by intrathecal glycine and related amino acids. Pharmacol
Biochem Behav 1988; 29: 73-8.

21. Bristow DR, Bowery NG, Woodruff GN: Light microscopic autoradiographic localisation of $\left[{ }^{3} \mathrm{H}\right]$ glycine and $\left[{ }^{3} \mathrm{H}\right]$ strychnine binding sites in rat brain. Eur J Pharmacol 1986; 126: 303-7.

22. Logan WJ, Snyder SH. High affinity uptake systems for glycine, glutamic and aspartic acids in synaptosomes of rat central nervous tissues. Brain Res 1972; 42: 413-31.

23. Pan ZH, Slaughter MM. Comparison of the actions of glycine and related amino acids on isolated third order neurons from the tiger salamander retina. Neuroscience 1995; 64: 153-64.

24. Sokal DM, Chapman V. Spinal GABA ${ }_{B}$-receptor antagonism increases nociceptive transmission in vivo. Neuroreport 2001; 12: 3247-50.

25. Hao JX, Xu XJ, Wiesenfeld-Hallin Z. Intrathecal $\gamma$-aminobutyric $\operatorname{acid}_{B}\left(G A B A_{B}\right)$ receptor antagonist CGP 35348 induces hypersensitivity to mechanical stimuli in the rat. Neurosci Lett 1994; 182: 299-302.

26. Moor KA, Kohno T, Karchewski LA, Scholz J, Baba H, Woolf CJ. Partial peripheral nerve injury promotes a selective loss of GABAergic inhibition in the superficial dorsal horn of the spinal cord. J Neurosci 2002; 22: 6724-31.

27. Hwang JH, Yaksh TL. The effect of spinal GABA receptor agonists on tactile allodynia in a surgically-induced neuropathic pain model in the rat. Pain 1997; 70: 15-22.

28. Gwak YS, Tan HY, Nam TS, Paik KS, Hulsebosch CE, Leem JW. Activation of spinal GABA receptors attenuates chronic central neuropathic pain after spinal cord injury. J Neurotrauma 2006; 23: 1111-24. 